\title{
Elasto-Dynamic Model-Based Control of Non-Redundant Cable-Driven Parallel Robots
}

\author{
Sana Baklouti ${ }^{1},{\text { Stéphane } \text { Caro }^{2} \text {, and Eric Courteille }}^{1}$ \\ 1 Univ Rennes, INSA Rennes, LGCGM-EA 3913, F-35000 Rennes, France \\ sana.baklouti, eric.courteille@insa-rennes.fr \\ 2 CNRS, Laboratoire des Sciences du Numérique de Nantes, UMR CNRS 6004, \\ Nantes, France \\ Stephane.Caro@ls2n.fr
}

\begin{abstract}
This paper deals with a model-based feed-forward torque control strategy of non-redundant cable-driven parallel robots (CDPRs). The proposed feed-forward controller is derived from an inverse elastodynamic model of the CDPR to compensate for the dynamic and oscillatory effects due to cable elasticity. A PID feedback controller ensures stability and disturbance rejection. Simulations confirm that tracking errors can be reduced by the proposed strategy compared to conventional rigid body model-based control.
\end{abstract}

\section{Introduction}

Cable-driven parallel robots (CDPRs) contain a set of flexible cables that connect a fixed frame to an end-effector (EE) with a coiling mechanism for each cable. They have been used in many applications like pick-and-place [1], rehabilitation [2], painting and sandblasting of large structures [3]. Thanks to their low inertia, CDPRs can reach high velocities and accelerations in large workspaces [4]. Several controllers have been proposed in the literature to improve CDPR accuracy locally or on trajectory tracking [5], [6], [7]. In [8], the control of CDPR in the operational space is presented, where the CDPR model is derived using Lagrange equations of motion for constrained systems, while considering non elastic but sagging cables through the Assumed Mode Method. In [9], a discrete-time control strategy is proposed to estimate the position accuracy of the EE by taking into account the actuator model, the kinematic and static behavior of the CDPR. Multiple papers deal with the problem of controlling CDPRs while considering cable elongations and their effect on the dynamic behavior. A robust $\mathrm{H}_{\infty}$ control scheme for CDPR is described in [10] while considering the cable elongations into the dynamic model of the EE and cable tension limits. A control strategy is proposed for CDPRs with elastic cables in [11], [12], [13]. It consists in adding an elongation compensation term to the control law of a CDPR with rigid cables, using singular perturbation theory. It requires the measurement of cables length and the knowledge of the EE pose real-time through exteroceptive measurements. 
Feed-forward model-based controllers are used to fulfillaccuracy improvement by using a CDPR reference model. This latter predicts the mechanical behavior of the robot; and then generates an adequate reference signal to be followed by the CDPR. This type of control provides the compensation of the desirable effects without exteroceptive measurements. A model-based control scheme for CDPR used as a high rack storage is presented in [14]. This research work takes into account the mechanical properties of cables, namely their elasticity. This strategy, integrating the mechanical behavior of cables in the reference signal, enhances the CDPR performances. However, it compensates for the EE positioning errors due to its rigid body behavior. The mechanical response of the robot is predicted when the mechanical behavior of the cables is not influenced by their interaction with the whole system, namely, the cable elongation is estimated. As a consequence, the main contribution of this paper deals with the coupling of a model-based feed-forward torque control scheme for CDPR with a PID feedback controller. The feed-forward controller is based on the elasto-dynamic model of CDPR to predict the full dynamic and oscillatory behavior of the CDPR and to generate the adequate reference signal for the control loop.

This paper is organized as follows: Section 2 presents the feed-forward modelbased control strategy proposed in this paper in addition to the existing rigid and elasto-static models. In Section 3, the proposed control strategy is implemented for a CDPR with three cables, a point-mass EE and three Degree-Of-Freedom (DOF) translational motions. Simulation results are presented to confirm the improvement of trajectory accuracy when using the proposed control strategy compared to conventional approaches. Conclusions and future work are drawn in Section 4.

\section{Feed-forward model-based controller}

The control inputs are mostly obtained by a combination of feed-forward inputs, calculated from a reference trajectory using a reference model of CDPR, and feedback control law, as in [15], [16]. The used control scheme shown in Fig. (1), is composed of a feed-forward block in which the inverse kinematic model is determined based on a CDPR reference model (Red block in Fig. (1)). This latter is a predictive model of the dynamic behavior of the mechanism. Its input is the motor torque vector $\boldsymbol{\zeta}_{r g} \in \mathbb{R}^{n}$, and its output is the reference winch rotation angle vectorq $q_{\text {ref }} \in \mathbb{R}^{n} ; n$ being the number of actuators.

The relationship between $\mathbf{q}_{\text {ref }}$ and the cable length vectorl $\mathbf{l}_{\text {ref }} \in \mathbb{R}^{n}$ is expressed as:

$$
\mathbf{l}_{r e f}-\mathbf{l}_{0}=\mathbf{R}\left(\mathbf{q}_{0}-\mathbf{q}_{r e f}\right),
$$

where $\mathbf{R} \in \mathbb{R}^{n \times n}$ is a diagonal matrix that is a function of the gear-head ratios and winch radius. $\mathbf{l}_{0} \in \mathbb{R}^{n}$ is the cable length vector at the static equilibrium and $\mathbf{q}_{0} \in \mathbb{R}^{n}$ is an offset vector corresponding to the cable length when $\mathbf{q}_{\text {ref }}=\mathbf{0}$. This offset is compensated at the rest time. The unwound cable length of the $i$ th cable is calculated using the CDPR inverse geometric model. 


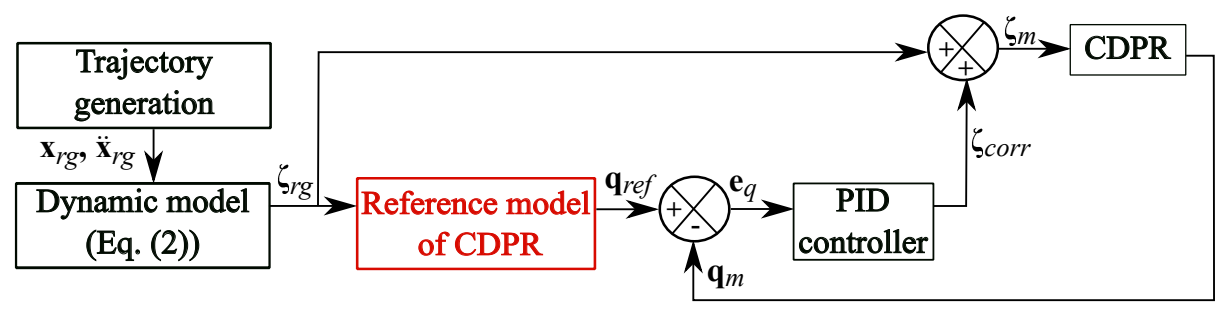

Fig. 1: Feed-forward model-based PID control

$\boldsymbol{\zeta}_{r g}$ is calculated using the following dynamic model of the CDPR, which depends on the desired EE pose $\mathbf{x}_{r g}$ and acceleration $\ddot{\mathbf{x}}_{r g}$ :

$$
\boldsymbol{\zeta}_{r g}=\mathbf{R} \boldsymbol{\tau}_{r g}, \quad \boldsymbol{\tau}_{r g}=\mathbf{W}_{r g}^{-1}\left(\mathbf{w}_{g}+\mathbf{w}_{e}-\mathbf{M} \ddot{\mathbf{x}}_{r g}\right),
$$

where $\boldsymbol{\tau}_{r g} \in \mathbb{R}^{n}$ is a set of positive tensions. $\mathbf{W}_{r g} \in \mathbb{R}^{m \times n}$ is the CDPR wrench matrix, $m$ being the DOF of its moving-platform. It is a function of $\mathbf{x}_{r g}$ and maps the EE velocities to the cable velocity vector. $\mathbf{M} \in \mathbb{R}^{m \times m}$ is the EE mass matrix, $\mathbf{w}_{g} \in \mathbb{R}^{m}$ is the wrench vector due to gravity acceleration and $\mathbf{w}_{e} \in \mathbb{R}^{m}$ denotes the external wrench.

In each drive, a feedback PID controller sets the vector of corrected motor torque $\boldsymbol{\zeta}_{\text {corr }} \in \mathbb{R}^{n}$. This latter is added to $\boldsymbol{\zeta}_{\text {rg }}$ to get the motor torque $\boldsymbol{\zeta}_{m} \in \mathbb{R}^{n}$, which results in the measured winch angular displacementsq $m \in \mathbb{R}^{n}$. The PID feedback control law is expressed as follows:

$$
\boldsymbol{\zeta}_{m}=\boldsymbol{\zeta}_{r g}+\mathbf{K}_{p} \mathbf{e}_{q}+\mathbf{K}_{d} \dot{\mathbf{e}}_{q}+\mathbf{K}_{i} \int_{t_{i}}^{t_{i}^{+}} \mathbf{e}_{q}(t) d t,
$$

where $\mathbf{K}_{p} \in \mathbb{R}^{n \times n}$ is the proportional gain matrix, $\mathbf{K}_{d} \in \mathbb{R}^{n \times n}$ is the derivative gain matrix, $\mathbf{K}_{i} \in \mathbb{R}^{n \times n}$ is the integrator gain matrix, $\mathbf{e}_{q}=\mathbf{q}_{\text {ref }}-\mathbf{q}_{m}$ is the error to minimize, leading to the correction torque vector:

$$
\boldsymbol{\zeta}_{\text {corr }}=\mathbf{K}_{p} \mathbf{e}_{q}+\mathbf{K}_{d} \dot{\mathbf{e}}_{q}+\mathbf{K}_{i} \int_{t_{i}}^{t_{i}^{+}} \mathbf{e}_{q}(t) d t .
$$

It is noteworthy that $\boldsymbol{\zeta}_{\text {corr }}$ depends on the CDPR reference model used to calculate the vector $\mathbf{q}_{r e f}$. To the best of our knowledge, two CDPR models have been used in the literature for the feed-forward model-based control of CDPRs with non-sagging cables: $(i)$ rigid model and (ii) elasto-static model. As a consequence, one contribution of this paper deals with the determination of the elasto-dynamic model of CDPRs to be used for feed-forward control.

\section{$2.1 \quad$ Rigid model}

The CDPR rigid model considers cables as rigid links. It is assumed that while applying the motor torque $\boldsymbol{\zeta}_{r g}$, the cables tension vector is equal to $\boldsymbol{\tau}_{r g}$ and the reference signal $\mathbf{q}_{\text {ref }}$ anticipates neither the cable elongation nor the oscillatory motions of the EE. The PID feedback controller uses the motor encoders response $\mathbf{q}_{m}$, which is related to the rolled or unrolled cable length $\mathbf{l}_{r g}$, which corresponds to the winch angular displacement $\mathbf{q}_{r g}$. It should be noted that the 
cable elongations and EE oscillatory motions are not detected here, and as a consequence, cannot be rejected.

\section{$2.2 \quad$ Elasto-static model}

The CDPR elasto-static model integrates a feed-forward cable elongation compensation [14]. It is about solving a static equilibrium at each EE pose while considering cable elasticity. Assuming that each cable is isolated, the cable elongation vector $\delta \mathbf{l}_{e s}$ is calculated knowing the cable tension vector $\boldsymbol{\tau}_{r g}$. The elastostatic cable tension vector $\boldsymbol{\tau}_{e s}$ is equal to $\boldsymbol{\tau}_{r g}$. The relationship between $\delta l_{e s}^{i}$ and $\tau_{e s}^{i}$ on the $i$ th cable takes the following form with a linear elastic cable model:

$$
\tau_{e s}^{i}=\tau_{r g}^{i}=E S \frac{\delta l_{e s}^{i}}{\delta l_{e s}^{i}+l_{r g}^{i}},
$$

where $E$ is the cable modulus of elasticity and $S$ is its cross-section area.

When $\mathbf{q}_{r g}$ is used as a reference signal in the feedback control scheme, the EE displacement $\delta \mathbf{x}_{e s}$ is obtained from cable elongation vector $\delta \mathbf{l}_{e s}$. To compensate for the cable elongation effects, $\delta \mathbf{l}_{e s}$ is converted into $\delta \mathbf{q}_{e s}$, which corrects the angular position $\mathbf{q}_{r g}$. Thus, the elasto-static reference angular displacement $\mathbf{q}_{r e f}^{e s}$ becomes:

$$
\mathbf{q}_{r e f}^{e s}=\mathbf{q}_{r g}-\delta \mathbf{q}_{e s} .
$$

As the CDPR cables tension is always positive, $\delta \mathbf{l}_{e s}>0$ corresponding to $\delta \mathbf{q}_{e s}<0$. The reference signal $\mathbf{q}_{r e f}^{e s}$ corresponds to a fake position of the EE for the cable elongation compensation. Here, under the effect of cable elongations, the reference EE pose is estimated to achieve the desired pose. Although the elasto-static reference model takes into account the cable elongations, the non-compensation for the EE pose errors due to the mechanism dynamic and elasto-dynamic behavior is not considered.

\subsection{Elasto-dynamic model}

The CDPR elasto-dynamic model takes into account the oscillatory and dynamic behavior of the $\mathrm{EE}$ due to cable elongations. Here, the cables are no-longer isolated and are affected by the EE dynamic behavior. Cable elongations make the EE deviate from its desired pose $\mathbf{x}_{r g}$. The real EE pose is expressed as: $\mathbf{x}_{e d}=\mathbf{x}_{r g}+\delta \mathbf{x}_{e d}$. The EE displacement leads to some variations in both cable lengths and cable tensions. Indeed, the $i$ th cable tension $\boldsymbol{\tau}_{e d}^{i}$ obtained from the elasto-dynamic model differs from $\tau_{r g}^{i}$ :

$$
\tau_{e d}^{i}=\tau_{r g}^{i}+\delta \tau_{e d}^{i}=E S \frac{\delta l_{e d}^{i}}{\delta l_{e d}^{i}+l_{r g}^{i}},
$$

where $\delta l_{e d}^{i}$ is the $i$ th cable elongation assessed by considering cable elasticity and oscillations. The CDPR elasto-dynamic model takes the form:

$$
\mathbf{M} \ddot{\mathbf{x}}_{e d}=\mathbf{W}_{e d} \boldsymbol{\tau}_{e d}+\mathbf{w}_{g}+\mathbf{w}_{e}
$$

where $\mathbf{W}_{e d}$ is CDPR wrench matrix expressed at EE pose $\mathbf{x}_{e d}$. Once $\mathbf{x}_{e d}$ and the cable tension vector $\boldsymbol{\tau}_{e d}$ are calculated, the cable elongation vector $\delta \mathbf{l}_{e d}$ can 
be determined. This latter is converted into $\delta \mathbf{q}_{\text {ed }}$, which corrects the angular position vector $\mathbf{q}_{r g}$. The reference angular displacement $\mathbf{q}_{r e f}^{e d}$ becomes:

$$
\mathbf{q}_{r e f}^{e d}=\mathbf{q}_{r g}-\delta \mathbf{q}_{e d} .
$$

The proposed control strategy based on the elasto-dynamic model leads to a feed-forward controller for EE oscillatory motion compensation in addition to the conventional rigid body feedback while considering the measurements from motor encoders. It should be noted that this feed-forward controller will not disrupt the rigid body feedback stability.

\section{Control of a spatial CDPR with a point-mass end-effector}

A spatial CDPR with three cables and three translational-DOF is considered in this section. This CDPR is composed of a point-mass EE, which is connected to three massless and linear cables. A configuration of the CREATOR prototype (Fig. (2a)), being developed at LS2N, is chosen such that the cables are tensed along a prescribed trajectory. The Cartesian coordinate vectors of the cable

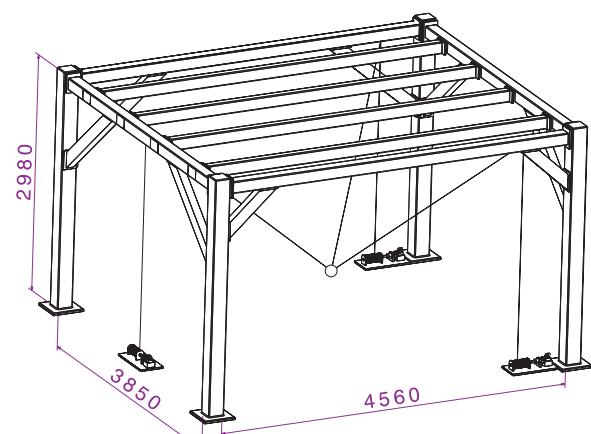

(a)

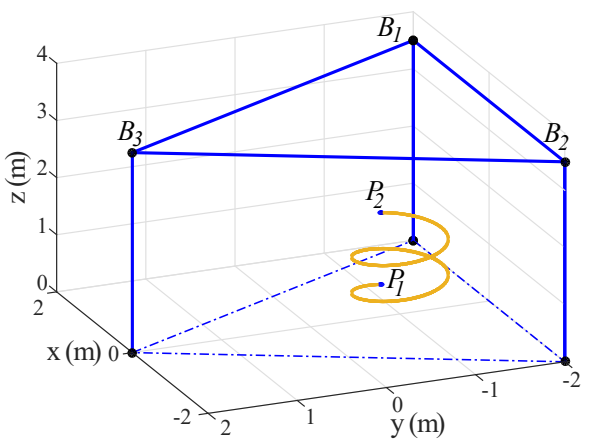

(b)

Fig. 2: (a) CREATOR prototype CAD diagram (b) End-effector desired path

exit points expressed in the global frame $\mathcal{F}_{b}$ are: $\mathbf{b}_{1}=\left[\begin{array}{ll}2.0,-2.0,3.5\end{array}\right]^{T} \mathrm{~m}$, $\mathbf{b}_{2}=[-2.0,-2.0,3.5]^{T} \mathrm{~m}, \mathbf{b}_{3}=[0.0,2.0,3.5]^{T} \mathrm{~m}$. The EE mass is equal to $20 \mathrm{~kg}$. The cables diameter is equal to $1 \mathrm{~mm}$. Their modulus of elasticity is equal to $70 \mathrm{GPa}$.

\subsection{Trajectory generation}

A circular helical trajectory, shown in Fig. (2b), is used from static equilibrium to steady state to evaluate the efficiency of the feed-forward model-based controller while considering three CDPR reference models. The EE moves from point $P_{1}$ of Cartesian coordinate vector $\mathbf{p}_{1}=\left[\begin{array}{ll}0.5, & -1.0,0.25\end{array}\right]^{T} \mathrm{~m}$ to point $P_{2}$ of Cartesian 
coordinate vector $\mathbf{p}_{2}=[0.5,-1.0,1.5]^{T} \mathrm{~m}$ along a circular helix. The latter is defined by the following equations:

$$
x(t)=R \cos \left(t_{\alpha}\right)+\beta_{0}, y(t)=R \sin \left(t_{\alpha}\right)+\beta_{1}, z(t)=p_{t} t_{\alpha}+\beta_{2},
$$

with

$$
t_{\alpha}=a_{5}\left(\frac{t}{t_{s i m}}\right)^{5}+a_{4}\left(\frac{t}{t_{s i m}}\right)^{4}+a_{3}\left(\frac{t}{t_{s i m}}\right)^{3}+a_{2}\left(\frac{t}{t_{s i m}}\right)^{2}+a_{1}\left(\frac{t}{t_{s i m}}\right)+a_{0} .
$$

The coefficients of the five-order polynomial $t_{\alpha}$ are chosen in such a way that the EE Cartesian velocities and accelerations are null at the beginning and the end of the trajectory. $R$ is the helix radius, $p_{t}$ is helix pitch. $\beta_{0}, \beta_{1}$ and $\beta_{2}$ are constants. Here, $a_{5}=24 \pi, a_{4}=-60 \pi, a_{3}=40 \pi, a_{2}=a_{1}=a_{0}=0, p_{t}=0.1 \mathrm{~m}$, $\beta_{0}=0.5 \mathrm{~m}, \beta_{1}=-1.0 \mathrm{~m}, \beta_{2}=0.25 \mathrm{~m}, R=0.5 \mathrm{~m}$ and $t_{\text {sim }}=15 \mathrm{~s}$. The velocity maximum value is $0.8 \mathrm{~m} / \mathrm{s}$. The acceleration maximum value is $1.2 \mathrm{~m} / \mathrm{s}^{2}$.

\subsection{Controller tuning}

The PID feedback controller is tuned using the Matlab ${ }^{3}$ PID tuning tool. This latter aims at finding the values of proportional, integral, and derivative gains of a PID controller in order to minimize error $\mathbf{e}_{q}$ and to reduce the EE oscillations. In the PID tuner work-flow, a plant model is defined from the simulation data, where the input is $\mathbf{e}_{q}$ and the output is $\boldsymbol{\zeta}_{\text {corr }}$. The gains obtained for the three control schemes are the following:

- Rigid model based: $K_{p}=3, K_{i}=1.5$ and $K_{d}=1.5$

- Elasto-static model based: $K_{p}=0.53, K_{i}=0.2$ and $K_{d}=0.18$

- Elasto-dynamic model based: $K_{p}=0.33, K_{i}=0.16$ and $K_{d}=0.15$

It is noteworthy that the gains decrease from the rigid model to the elasto-dynmic reference model.

\subsection{End-effector position errors}

The EE position error is defined as the the difference between its desired position $\mathbf{x}_{r g}$ and its real one. This latter should be normally determined experimentally. As experimentations are not yet done, a good CDPR predictive model should be used to estimate the EE real pose. The CDPR elasto-dynamic model is the closest to the real CDPR with non sagging cables; so, it is used to predict the real behavior of the CDPR. The input of this model is $\zeta_{m}$, which leads to $\mathbf{x}_{e d}^{m}$. The position error is defined as $\delta \mathbf{p}=\mathbf{x}_{r g}-\mathbf{x}_{e d}^{m}$. To analyze the relevance of the proposed control strategy, the three control schemes under study were simulated through Matlab-Simulink ${ }^{\circledR}$. Figure (3a) shows the norm of the EE position error $\|\delta \mathbf{p}\|$ when the proposed feed-forward control law is applied while using successively the three CDPR models to generate the reference signal.

Figure (3b) illustrates the EE position error along the $z$-axis $\delta z$, which is the main one as the CDPR under study is assembled in a suspended configuration. The red (gree, blue, resp.) curve depicts the EE position error when the elastodynamic (elasto-static, rigid, resp.) model is used as a reference. The root-meansquare (RMS) of $\delta \mathbf{p}$ is equal to $8.27 \mathrm{~mm}$ when the reference signal is generated

\footnotetext{
${ }^{3}$ www.mathworks.com/help/slcontrol/ug/introduction-to-automatic-pid-tuning.html
} 


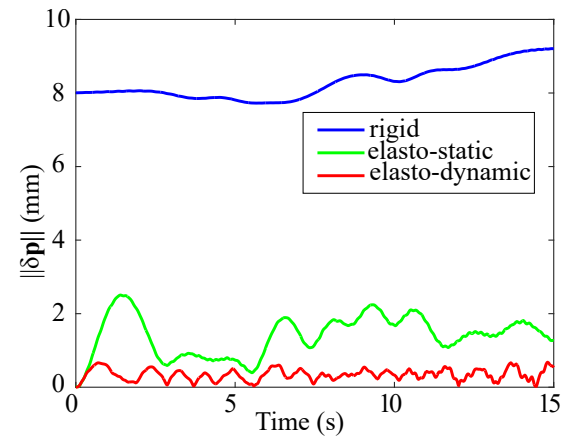

(a)

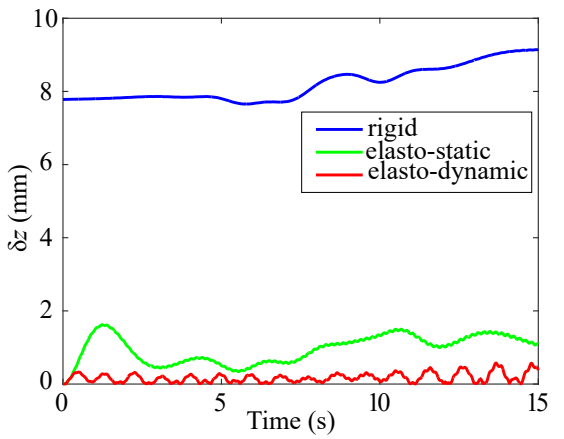

(b)

Fig. 3: (a) Position error norm (b) Position error along z-axis of the end-effector

by the rigid model of CDPR. This value is equal to $1.41 \mathrm{~mm}$ when the elastostatic model is used. This represents a maximum relative difference of $17 \%$ in terms of EE tracking errors. When the elasto-dynamic model is used to generate the reference signal of control, the EE position error RMS decreases to $0.31 \mathrm{~mm}$, which represents a difference of $24 \%$ in comparison to the position errors when adopting the elasto-static model.

As shows the histogram in Fig. (4), the position error of the EE is dominated by the vertical direction regardless of the used reference model. Tracking errors and settling times are reduced significantly while the elasto-dynamic feed-forward control compared to classical feed-forwards.

\section{Conclusions and future work}

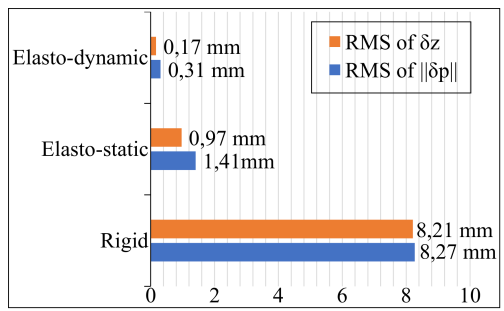

Fig. 4: Histogram of the RMS of $\|\delta \mathbf{p}\|$ and $\delta z$

This paper proposed a model-based feed-forward control strategy for non-redundant CDPRs. An elasto-dynamic model of CDPR was proposed to anticipate the full dynamic behavior of the mechanism. Accordingly, a contribution of this paper deals with a good simulation model of the CDPR, including the vibratory effects, cable elongations and their interaction with the whole system, used as a reference control model. The comparison between the position errors obtained when using the proposed elasto-dynamic model or the classical rigid and elasto-static ones as control references shows meaningful differences. These differences reveal that the proposed control strategy guarantees a better trajectory tracking when adopting the proposed elasto-dynamic model to generate the reference control signal for a non-redundant CDPR. Experimental validations will be carried later on. Future work will also deal with the elasto-dynamic model-based control of redundant actuated CDPRs. 


\section{References}

1. Dallej, T., Gouttefarde, M., Andreff, N., Michelin, M., Martinet, P. (2011, September). Towards vision-based control of cable-driven parallel robots. In Int. Conf. on Intelligent Robots and Systems (IROS), 2011 IEEE/RSJ (pp. 28552860). IEEE.

2. Hernandez, E., Valdez, S. I., Carbone, G., Ceccarelli, M. (2017, October). Design Optimization of a Cable-Driven Parallel Robot in Upper Arm TrainingRehabilitation Processes. In International Symposium on Multibody Systems and Mechatronics (pp. 413-423). Springer, Cham.

3. Gagliardini, L., Caro, S., Gouttefarde, M., Girin, A. (2016). Discrete reconfiguration planning for cable-driven parallel robots. Mechanism and Machine Theory, 100, 313-337.

4. Lamaury, J., Gouttefarde, M., Chemori, A., Herv, P. E. (2013, November). Dualspace adaptive control of redundantly actuated cable-driven parallel robots. In Intelligent Conference on Robots and Systems (IROS), 2013 IEEE/RSJ (pp. 4879-4886). IEEE.

5. Jamshidifar, H., Fidan, B., Gungor, G., Khajepour, A. (2015). Adaptive Vibration Control of a Flexible Cable Driven Parallel Robot. IFAC-PapersOnLine, 48(3), 1302-1307.

6. Zi, B., Duan, B. Y., Du, J. L., Bao, H. (2008). Dynamic modeling and active control of a cable-suspended parallel robot. Mechatronics, 18(1), 1-12.

7. Pott, A., Mütherich, H., Kraus, W., Schmidt, V., Miermeister, P., Verl, A. (2013). IPAnema: a family of cable-driven parallel robots for industrial applications. In Cable-Driven Parallel Robots (pp. 119-134). Springer Berlin Heidelberg.

8. Cuevas, J. I. A., Laroche, E., Piccin, O. (2018). Assumed-Mode-Based Dynamic Model for Cable Robots with Non-straight Cables. In Cable-Driven Parallel Robots (pp. 15-25). Springer, Cham.

9. Merlet, J. P. (2017). Simulation of Discrete-Time Controlled Cable-Driven Parallel Robots on a Trajectory. IEEE Trans. on Robotics.

10. Laroche, E., Chellal, R., Cuvillon, L., Gangloff, J. (2013). A Preliminary Study for $\mathrm{H}_{\infty}$ Control of Parallel Cable-Driven Manipulators. In Cable-Driven Parallel Robots (pp. 353-369). Springer Berlin Heidelberg.

11. Khosravi, M. A., Taghirad, H. D. (2014). Dynamic modeling and control of parallel robots with elastic cables: singular perturbation approach. IEEE Transactions on Robotics, 30(3), 694-704.

12. Khosravi, M. A., Taghirad, H. D. (2016). Stability analysis and robust PID control of cable driven robots considering elasticity in cables. AUT Journal of Electrical Engineering, 48(2), 113-126.

13. Khosravi, M. A., Taghirad, H. D. (2011). Dynamic analysis and control of cable driven robots with elastic cables. Transactions of the Canadian Society for Mechanical Engineering, 35(4), 543-558.

14. Bruckmann, T., Lalo, W., Sturm, C., Schramm, D., Hiller, M. (2013). Design and realization of a high rack and retrieval machine based on wire robot technology.

15. Lamaury, J., Gouttefarde, M. (2013, May). Control of a large redundantly actuated cable-suspended parallel robot. In Int. Conf. on Robotics and Automation (ICRA), 2013 IEEE (pp. 4659-4664).

16. Bayani, H., Sadeghian, R., Masouleh, M. T., Kalhor, A. (2015, October). On the control of planar cable-driven parallel robot via classic controllers and tuning with intelligent algorithms. In Int. Conf. on Robotics and Mechatronics (ICROM), 2015 3rd RSI (pp. 623-628). 